\title{
Intelligent Control of Thermal Comfort in Automobile
}

\author{
Yadollah Farzaneh, Ali Akbarzadeh Tootoonchi \\ Department of Mechanical Engineering \\ Ferdowsi University of Mashhad \\ Mashhad, IRAN \\ ya_farzaneh@yahoo.com, ali_akbarzadeh_t@yahoo.com
}

\begin{abstract}
Saving consumable energy and maintaining the thermal comfort level are two main topics in heating, ventilating and air conditioning (HVAC) control field. A controller with temperature feedback can not best achieve these goals. Fanger's "Predicted Mean Vote" (PMV) index is commonly used to better describe comfort. Therefore, this paper proposes a simpler form of PMV index for using as fuzzy controller feedback. A fuzzy controller with PMV feedback is first designed for automobile climate control. Next, the membership functions of the fuzzy controller are optimized by Genetic Algorithm. Finally, robustness analysis is performed to indicate the robustness of optimized controller to variable variation. Results show that the proposed controller not only better controls the thermal comfort but it also reduces energy consumption.
\end{abstract}

Keywords-climate control, passenger comfort, PMV, robustness analyzes, Genetic Algorithm.

\section{INTRODUCTION}

Since an automobile is operated in various weather conditions, such as scorching heat and downpours, passenger thermal comfort is constantly affected by environmental changes. An air conditioning system must maintain an acceptable thermal comfort inside the cabin despite these changes. However, an air conditioning system inevitably uses energy, which increases automobile fuel consumption. This energy must be minimized. Therefore, an effective control procedure is needed to resolve the contradictions of low energy consumption and a pleasant driving climate.

Thermal comfort is a non-linear result of the interaction between environmental and personal factors as well as subjective feelings [1]. The most important environmental factors are: air temperature, mean radiant temperature, air velocity and relative humidity. Personal factors include activity level and clothing insulation.

In most articles air temperature is used as the controller feedback for automobile climate control [2, 3, 4]. It is clear that the air temperature alone is not sufficient for evaluating thermal comfort in the cabin [5]. PMV is a thermal comfort index which combines the above factors to a single value to indicate thermal sensation. Therefore, in this paper PMV index [5] is used to predict the thermal comfort inside the cabin.

PMV is simplified for an automobile cabin. It is used as a feedback for a fuzzy controller. Blower power and the position of air temperature door are adjusted by the controller to achieve comfort inside the cabin. An index for energy consumption is also suggested. Finally, the membership functions of fuzzy controller are tuned by Genetic Algorithm to further improve the performance of fuzzy controller. After optimization, robustness analysis is performed to show the robustness of optimized controller to variation in.

\section{THERMAL MODEL OF AUTOMOBILE CABIN}

Fig. 1 shows an overview of the air-conditioning system under study. Outside air or recirculated air is drawn into the air conditioner. It is first cooled by heat exchange within the evaporator. Then, it flows in two directions based on the position of the temperature door. One part flows as cool air, while the other is reheated within the heater core. Thus, the temperature door adjusts the amount of air to be reheated. The position of temperature door is adjusted by the fuzzy controller. The cool and warm air are remixed and blown into the automobile compartment through outlets. Blower power which is controlled by the fuzzy controller determines the flow rate of incoming air.

Mathematical model of the system is obtained from [6]. After modification, it is represented by (1).

$$
\begin{aligned}
A \cdot \dot{T}_{c a b i n} & +B \cdot T_{c a b i n}=C \cdot T_{a m b} \\
& +D \cdot T_{e R, i}+E \cdot T_{w, i}+F
\end{aligned}
$$




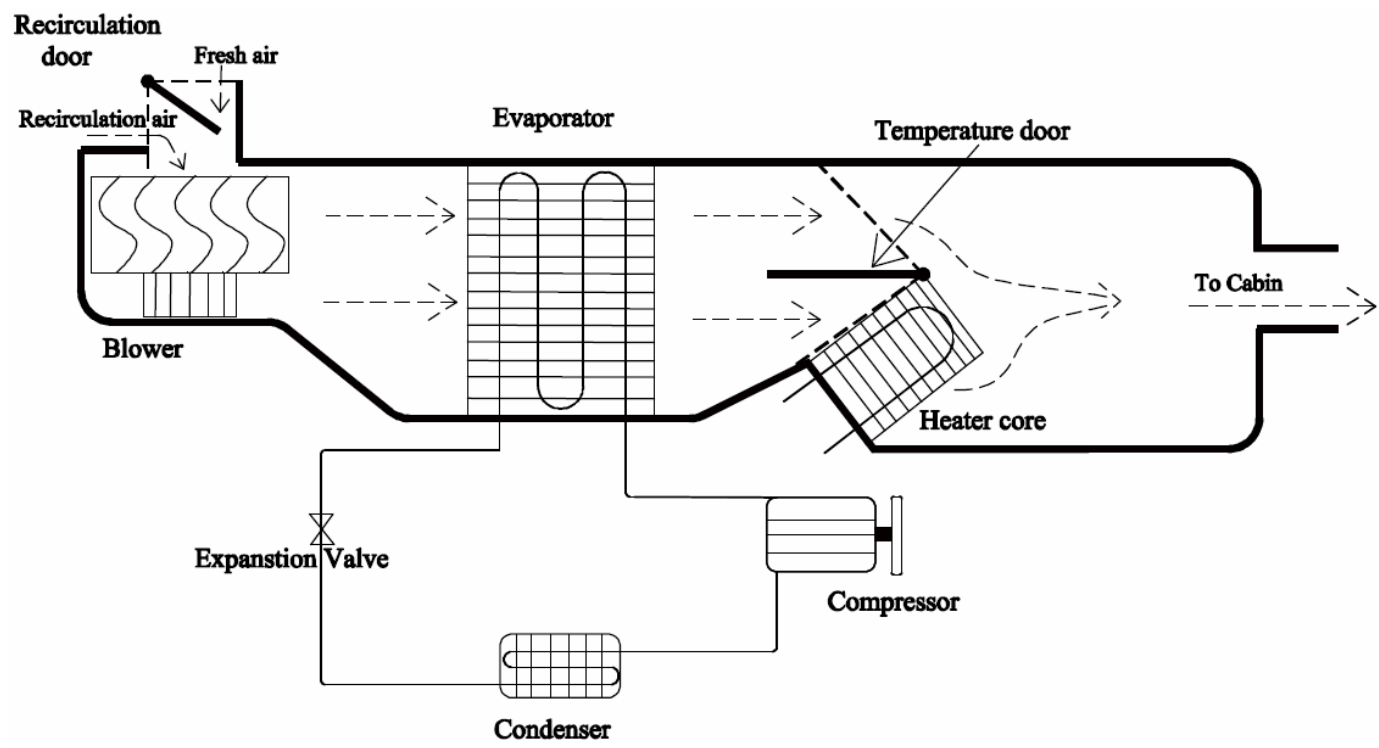

Figure 1. Schematic of automobile cabin and air conditioning systems

$$
\begin{aligned}
& A=\rho_{\text {air }} V_{\text {cabin }} \dot{m}_{\text {air }} C p_{a}{ }^{2} \\
& B=\dot{m}_{\text {air }} C p_{a}\left\{\dot{m}_{\text {air }} C p_{a}-(1-p f)\left(1-\varepsilon_{e}\right)\right. \\
& \left.\left[\dot{m}_{\text {air }} C p_{a}+(1-p t d) \varepsilon_{H} C_{\text {min, }}\right]+0.1202\right\} \\
& C=p f\left(1-\varepsilon_{e}\right) \dot{m}_{a i r} C p_{a}\left[\dot{m}_{a i r} C p_{a}+\right. \\
& \left.(1-p t d) \varepsilon_{H} C_{\min , H}\right]+\dot{m}_{a i r} C p_{a} 0.1202 \\
& D=\dot{m}_{\text {air }} C p_{a} \varepsilon_{e}\left[\dot{m}_{\text {air }} C p_{a} p t d+\right. \\
& \left.(1-p t d)\left(\dot{m}_{\text {air }} C p_{a}+\varepsilon_{H} C_{\min , H}\right)\right] \\
& E=-\varepsilon_{e}\left[\dot{m}_{\text {air }} C p_{a} p t d+(1-p t d)\right. \\
& \left.\left(\dot{m}_{a i r} C p_{a}+\varepsilon_{H} C_{\min , H}\right)\right](1-p t d) \varepsilon_{H} C_{\min , H} \\
& F=\dot{m}_{\text {air }} C p_{a} 0.2618 \\
& \mathrm{~V}_{\text {air }}=0.0245 \exp (4.0329 * \mathrm{bp})
\end{aligned}
$$

\section{CONTROLLING THE THERMAL COMFORT INSIDE THE CABIN}

Fuzzy logic has been successfully applied to controlling problems. The key for success was the ability of fuzzy systems to incorporate human expert knowledge. Fuzzy logic is naturally nonlinear and vague, thus it is suitable for systems with this behavior. Automobile air conditioning system is also nonlinear and complex. It is difficult for conventional methods to control it well [7] therefore fuzzy control is a good choice for controlling this system.

As stated before, temperature does not best describe the thermal comfort and therefore controller with temperature feedback cannot achieve desired comfort. A better solution is to use controller with PMV feedback as shown in Fig. 2.

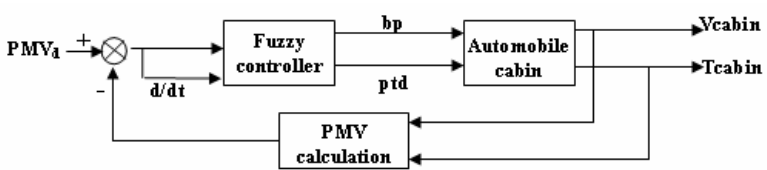

Figure 2. Controller with PMV feedback

PMV is next simplified by assuming air temperature and air velocity are the only two variables while the remaining four variables are assumed constant. With this simplification PMV will vary from -0.6 to +0.3 which is still within the comfort zone and therefore it is an acceptable prediction of comfort obtained.

Triangular fuzzy sets are chosen for this controller and are divided equally in two limits (Table I). Fuzzy rules for each controller outputs are indicated in Table II.

TABLE I. SUGGESTED INTERVAL FOR VARIABLES

\begin{tabular}{lll}
\hline & MIN & MAX \\
\hline PMV error & -3.5 & 3.5 \\
PMV error_dot & -1.5 & 1.5 \\
$\begin{array}{l}\text { Blower power (bp) } \\
\begin{array}{l}\text { Position of temperature door } \\
\text { (ptd) }\end{array}\end{array}$ & -0.1 & 1.33 \\
\hline
\end{tabular}

TABLE II. FUZZY RULES

\begin{tabular}{|l|l|l|l|}
\hline e_dot e & Negative & Zero & Positive \\
\hline Negative & High & High & Medium \\
\hline Zero & High & Low & Low \\
\hline Positive & High & Low & Low \\
\hline
\end{tabular}




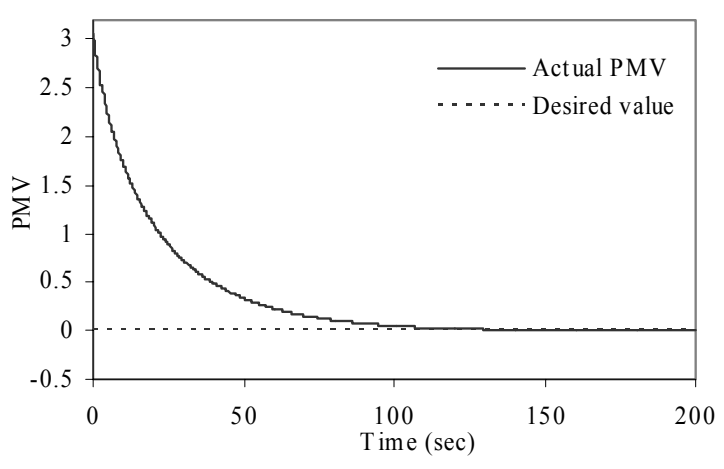

Figure 3. PMV variation in cabin

Simulation is performed assuming $35^{\circ} \mathrm{C}$ outside temperature environment and zero fresh air entering the system. Result of the fuzzy controller is shown in Fig. 3.

Minimal energy consumption is another performance criterion used in this study. It means that the compressor power should be minimized. COP relates the evaporator cooling capacity to the compressor power [8]. Therefore, evaporator cooling capacity can be used as a good index for compressor power and it should be as low as possible (Fig.4).

\section{TUNING FUZZY CONTROLLER THROUGH GENETIC ALGORITHM}

Fuzzy systems design can be divided into three main stages; namely, the selection of a proper set of input and output variables and their related universes of discourse, the identification of a suitable structure for the fuzzy system and the extraction of an optimal fuzzy rule-base. The most crucial task in fuzzy systems design is the formation of proper membership function with fuzzy rules. This directly affects the overall performance of the system.

If no primary knowledge is available, formation and tuning of membership functions is not an easy task. Furthermore, fuzzy systems do not have ability to learn [9] and complementary methods such as: artificial neural network and evolutionary algorithms should be used for Identification of fuzzy system parameters.

Recently, evolutionary algorithms and especially genetic algorithm are proposed as an instrument to optimize fuzzy systems. Genetic Algorithm (GA) is general and a global optimization method which is based on the natural evolution theory. GA is suitable for not well defined as well as irregular search spaces.

Optimization problem is defined as the objective function. Variables are coded as chromosomes and each chromosome is an answer to the problem. Fitness is assigned to each chromosome with respect to the objective function. Fittest chromosomes generate the next population through reproduction, crossover and mutation.

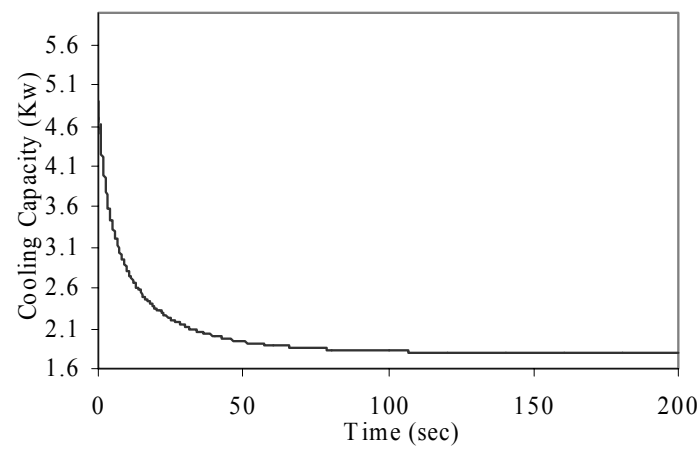

Figure 4. Evaporator cooling capacity

\section{A. General procedure for tuning fuzzy controller}

Important parameters such as membership functions and fuzzy rules are coded to chromosomes. Fitness value is given to each chromosome based on performance of fuzzy controller. Next, GA operators generate the next generation of population. This process continues until the desired performance is achieved or the number of generation exceeds a specific number (Fig. 5).

\section{B. Tuning the membership functions}

If the rule base is small the if-then structure of fuzzy rule is easy to understand and build with priori knowledge. The state evaluation fuzzy control rule and/or state trajectory approach can be used for defining fuzzy rules [10]. The control goals can be easily obtained by adjusting the membership functions. The formation of acceptable fuzzy membership functions is a subjective and time consuming task, thus GA is used to search and optimize the membership functions. In this study, the minmax limits of the membership functions are defined and GA finds the best limit for each input-output variable. Min-Max limits are normalized to $(0,1)$ and then coded to chromosomes. Controller has two inputs (PMV, PMV-dot) and two outputs (blower power, position of temperature door), thus the length of each chromosome is equal to eight Genes (Fig. 6).

Achieving comfort is the desired response of fuzzy controller. Initially GA is applied to optimize PMV without considering energy consumption. Zero value for PMV indicates optimum thermal comfort. Absolute summation of the differences between calculated and zero PMV during simulation is selected as the objective function for GA. Therefore GA attempts to minimize this difference by adjusting the membership functions.

$$
\text { Fitness function } 1=\sum|P M V-0|
$$

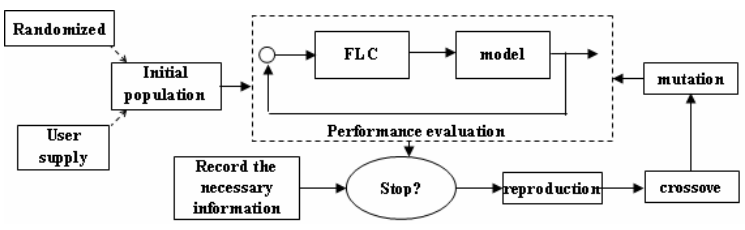

Figure 5. General GA optimization process 


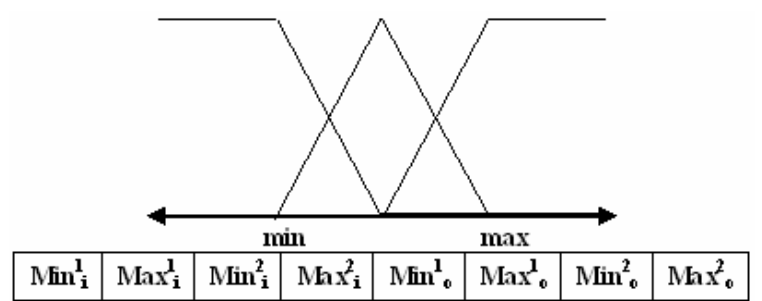

Figure 6. Chromosome coding

Results as indicated in Figs. 7, 8 show thermal comfort is achieved faster with the GA-tuned parameters, however, energy consumption is increased. Clearly, this increase in energy consumption is not acceptable.

Minimizing energy consumption while achieving optimum PMV is next considered. As indicated before a good index for compressor power and energy consumption is evaporator cooling capacity. The summation of evaporator cooling capacity during each simulation is the index used for energy consumption. These two goals result in a two-objective optimization problem, i.e., minimizing both the comfort error and energy consumption. The multi-objective goal can be converted into a single objective problem. One way to combine these goals is by applying proper weights to each goal.

\section{Fitness function $2=$}

$$
w_{1} \cdot \sum|P M V-0|+w_{2} \cdot \sum Q_{r}
$$

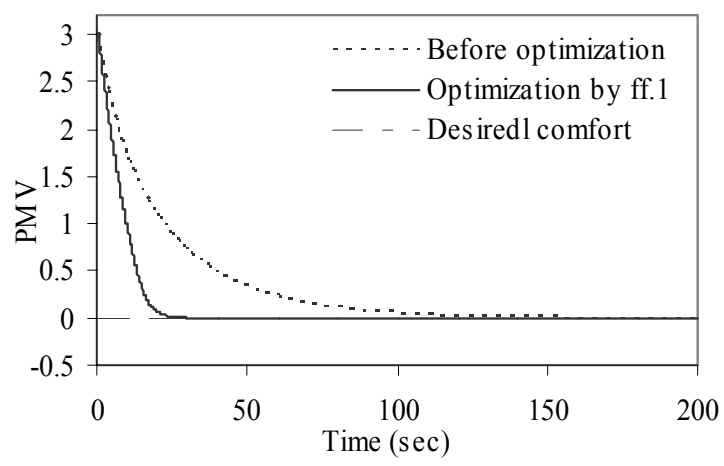

Figure 7. PMV in cabin

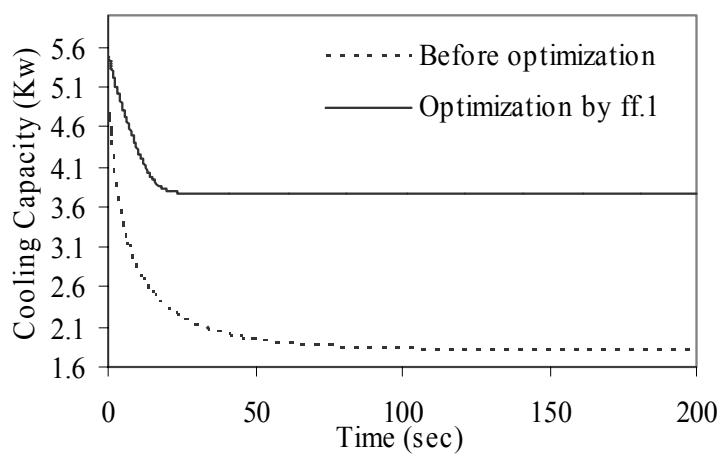

Figure 8. Evaporator cooling capacity
TABLE III. TUNED LIMITS OF VARIABLES

\begin{tabular}{lll}
\hline & MIN & MAX \\
\hline PMV error & -0.7353 & 2.6017 \\
PMV error_dot & -4.8533 & 0.2354 \\
Blower power & -0.7013 & 1.7729 \\
Position of temperature door & 0.3116 & 1.6376 \\
\hline
\end{tabular}

The optimized limits which minimize the objective function (Equation (4)) are listed in Table III.

Results of fuzzy controllers before and after optimization with fitness function-1 and fitness function- 2 are shown in Fig. 9.

As shown in Fig. 9 the optimized fuzzy with FF-2 reaches thermal comfort a bit later than the controller with FF-1. However, this slight delay in reaching comfort is well compensated by the significant reduction in fuel consumption. It can be concluded that optimization using FF-2 reaches thermal comfort while reducing energy consumption.

\section{ROBUSTNESS ANALYSIS}

Automotive cabin is affected by many random and uncontrollable parameters. Variation in uncontrolled parameters is one of the most important sources affecting system performance. These are part of the system model but are not under designer control and may strongly influence the behavior of the system. A good controller must be robust to changes in these variables.
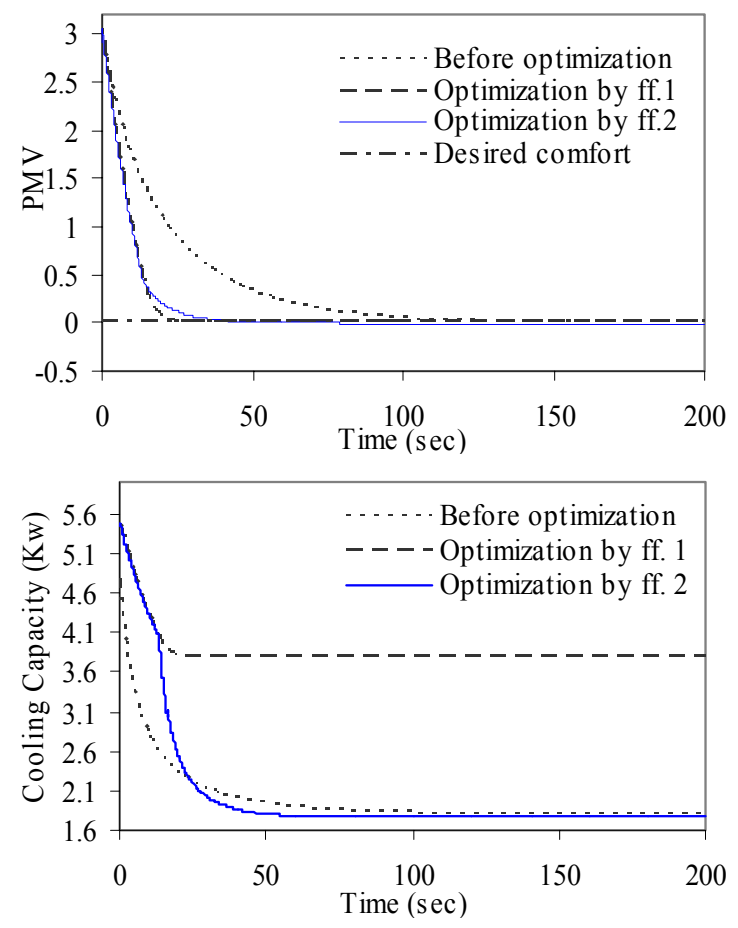

Figure 9. Fuzzy controller before and after optimization 


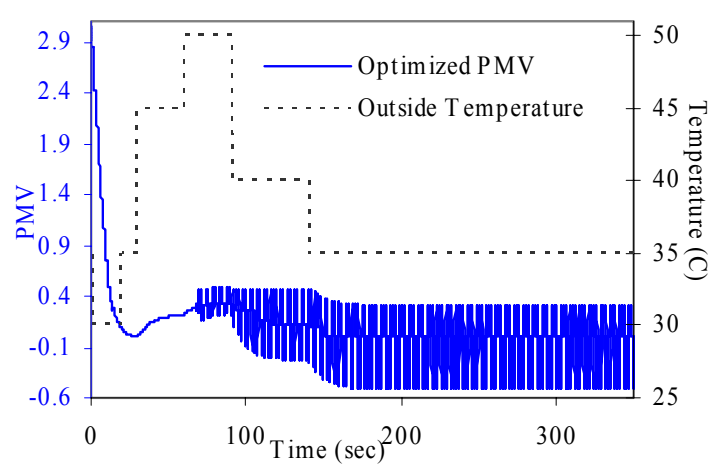

Figure 10. PMV during temperature variation

Optimization and robustness often work against each other. Therefore, robustness analysis should be performed after optimization to insure the effectiveness of controller over a wide range of conditions. Robustness is a characteristic of controller which minimizes the effect of uncertainty or variation in system parameters without eliminating the source of the uncertainty or variation.

In this study, thermal load is the most important uncontrollable parameter that affects system behaviour. Thermal load is affected by variations such as outside air temperature, sun radiation, opening the automotive windows and doors, ratio of fresh air to re-circulated air. Controller must be robust to change in these parameters.

To show the controller robustness we assume that the outside air temperature, an uncontrolled parameter, varies during the simulation while other conditions and parameters remain unchanged.

The controller is optimized by fitness function 2 . The weights are manually selected as $w 1=6$ and $w 2=1.2$. This produces equal effect for both cooling power as well as PMV error. Fig. 10 shows the PMV variation during the simulation.

As shown in Fig. 10 the controller is not robust and has unacceptable oscillation level. The fitness function should be changed to overcome this problem. Therefore, weights are selected as $\mathrm{w} 1=9$ and $\mathrm{w} 2=1$.

As can be seen from Fig. 11 the controller response during temperature changes is quite reasonable and quickly brings PMV to zero value. Therefore, the optimized controller is robust to parameter variations. The response of the controller before optimization is also shown. It is clear that this controller is also robust to temperature variation.

\section{CONCLUSION}

Controlling the heating, ventilating and air conditioning (HVAC) in the automobile cabin is a multi-criterion control problem. There is always being a trade-off to be made between occupant thermal comforts and efficient energy consumption. Human comfort is a complex reaction, involving physical, biological, and psychological responses to the given conditions. Thermal comfort can not be described with temperature alone. There are many parameters that affect thermal comfort such as,

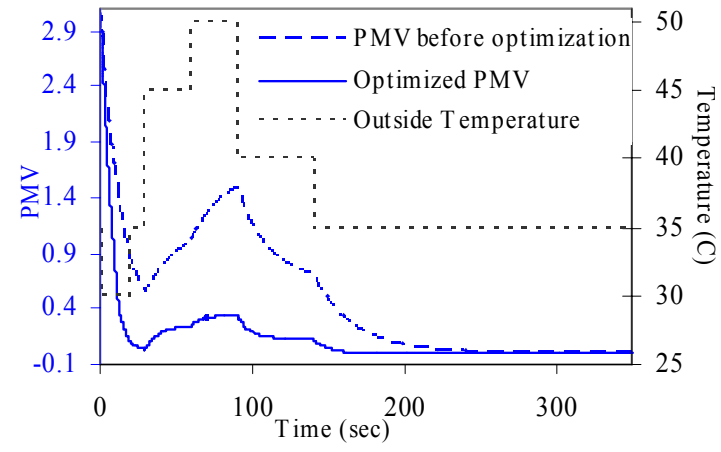

Figure 11. PMV during temperature variation

cabin air temperature, relative humidity, air velocity, environment radiation, activity level of passengers and clothing insulation. PMV index which combines the above parameters is used to indicate thermal comfort in cabin. A simplified form of PMV is introduced where temperature and air speed are the only two variables.

This paper presents a fuzzy controller for HVAC system of an automobile cabin. This controller uses thermal comfort instead of temperature as its feedback.

Preparing thermal comfort while minimizing energy consumption was the next goal of the controller. Genetic algorithm is used to improve the performance of fuzzy controller with respect to these goals. It is shown that after optimization controller reaches thermal comfort quicker while minimizing energy consumption. Finally, robustness analysis is performed to show the robustness of optimized controller to variations in system variables. Outside air temperature is varied and controller response is measured. Results indicate controller performs well to temperature variations.

\section{REFERENCES}

[1] P. O. Fanger, Thermal Comfort, Analysis and Applications in Environmental Engineering, McGraw Hill, 1972.

[2] T. Tabe, K. Matsui, T. Kakehi, and M. Ohba, "Automotive Climate Control,” IEEE Control Systems Magazine, pp. 20-24, 1986.

[3] K. C. Wei, and G.A. Dage, "An Intelligent Automotive Climate Control System,” pp. 2977-2982, 1995.

[4] H. Zhong et al., "Controlling indoor air temperature in a automobile with fuzzy controller via air-conditioning system," Shanghai Jiaotong Daxue Xuebao, pp. 1163-1166, 2001.

[5] ASHRAE handbook-fundamentals, Chap. 8, Thermal comfort, 2001.

[6] Y. Farzaneh, Intelligent control of automobile HVAC with fuzzy logic, M.S thesis, Ferdowsi university of Mashhad, Iran (in Persian), 2007.

[7] L. I. Davis, T. F. Sieja, R.W. Matteson, G.A. Dage, and R. Ames, "Fuzzy Logic for Vehicle Climate Control," 3rd IEEE Int'1. Conf. on fuzzy systems, pp. 530-534, 1994.

[8] J. Jabardo, W. G. Mamani, and M. R. Ianella. "Modeling and experimental evaluation of an automotive air conditioning system with variable capacity compressor,” Int. J Refrig. pp. 1157-73, 2003.

[9] K. H. Lee, First course on fuzzy theory and application, Springer, 2005.

[10] K. Tanaka, An Introduction to Fuzzy Logic for Practical Applications, Springer, 1997. 